\title{
Sex-Specific Analysis Is Lacking in Abstracts Presented at Arthroscopy Association of North America and American Orthopaedic Society for Sports Medicine Annual Meetings From 2016 to 2019
}

\author{
Carrie Huang, B.S., Arianna L. Gianakos, D.O., Meghan Merklein, M.D., \\ Angelica Pinninti, M.D., Brett D. Owens, M.D., and Mary K. Mulcahey, M.D.
}

\begin{abstract}
Purpose: To evaluate the presence of sex-specific analysis (SSA) in abstracts accepted for podium presentation at the Arthroscopy Association of North America (AANA) and American Orthopaedic Society for Sports Medicine (AOSSM) annual meetings from 2016 to 2019. Methods: Abstracts accepted for podium presentation at the AANA and AOSSM annual meetings from 2016 to 2019 were selected for review. Studies that included sex as a variable in a multifactorial statistical model were considered to have performed adequate SSA. Secondary data collected included whether the abstract had a female lead or senior author, the degrees of the female authors, and the anatomic focuses of studies with SSA. Results: Of the 891 total abstracts accepted for podium presentation at the AANA and AOSSM annual meetings from 2016 to $2019,90(10 \%)$ included SSA. There were 284 AANA abstracts, $24(8 \%)$ of which reported SSA. Of the 607 AOSSM abstracts, $66(11 \%)$ reported SSA. There were 43 female first authors $(15 \%)$ and 33 female senior authors $(12 \%)$ of the AANA abstracts compared with 92 female first authors (15\%) and 39 female senior authors $(6 \%)$ of the AOSSM abstracts. Of the 891 total abstracts, $135(15 \%)$ listed a female lead author and $72(8 \%)$ had a female senior author. Of the 135 female first authors, $92(68 \%)$ had an M.D., whereas 40 of the 72 female senior authors $(56 \%)$ had an M.D. Analysis of all abstracts combined showed a positive correlation between SSA and a female first author (Pearson correlation coefficient $=0.035, P=.147$ ), as well as between SSA and a female senior author (Pearson correlation coefficient $=0.052$, $P=.059)$. Conclusions: From 2016 to 2019 , only $10 \%$ of abstracts accepted for podium presentation at the AANA and AOSSM annual meetings included SSA. Altogether, women represented $15 \%$ of first authors and $8 \%$ of senior authors. Clinical Relevance: This study highlights the low percentage of SSA in abstracts presented at the AANA and AOSSM annual meetings from 2016 to 2019. Future studies should attempt to perform SSA when relevant to better evaluate differences in outcomes between male and female patients.
\end{abstract}

$\mathbf{P}$ revious literature has described sex differences in patients with sports injuries. ${ }^{1}$ Understanding these differences is important to better manage and treat athletes individually. It has been well established within the literature that there are differences between male and female athletes with various conditions including anterior cruciate ligament rupture, lower-extremity stress fractures, overuse injuries, and the development of metabolic conditions such as osteoporosis. ${ }^{1-3}$

From Tulane University School of Medicine, New Orleans, Louisiana, U.S.A. (C.H., M.K.M.); Jersey City Medical Center, Jersey City, New Jersey, U.S.A. (A.L.G., M.M.); Lewis Katz School of Medicine at Temple University, Philadelphia, Pennsylvania, U.S.A. (A.P.); and Warren Alpert Medical School of Brown University, Providence, Rhode Island, U.S.A. (B.D.O.).

The authors report no conflicts of interest in the authorship and publication of this article. Full ICMJE author disclosure forms are available for this article online, as supplementary material.

Received January 31, 2020; accepted September 12, 2020.
Anatomic, hormonal, biomechanical, and neuromuscular differences have all been found to contribute to the differences observed between men and women. Therefore, it is important to adequately analyze for sexrelated differences to better treat both male and female athletes.

Women were historically excluded from clinical research until 1993, when the National Institutes of Health (NIH) passed the Revitalization Act, which

Address correspondence to Mary K. Mulcahey, M.D., Tulane University School of Medicine, 1430 Tulane Ave, Box 8632, New Orleans, LA 70112, U.S.A.E-mail:mary.mulcahey.md@gmail.com

(C) 2020 THE AUTHORS. Published by Elsevier Inc. on behalf of the Arthroscopy Association of North America. This is an open access article under the CC BY-NC-ND license (http://creativecommons.org/licenses/by-nc-nd/4.0/).

2666-061X/2086

https://doi.org/10.1016/j.asmr.2020.09.007 
mandated the inclusion of women and minorities in NIH-funded clinical research. ${ }^{4}$ This statute states that any clinical trial including women as subjects must provide valid analysis of whether the variables being studied affect women differently than other subjects in the trial. However, it also states that inclusion is not required if there are "substantial scientific data" showing that there is no significant difference in the effects that the variables studied in the trial have on women. Additionally, if previous literature on the subject shows no significant sex differences, then sexspecific analysis (SSA) is not required. If prior studies neither support nor negate significant differences, then phase III clinical trials must include demographic characteristics, but they are not required to provide high statistical power for sex or gender comparisons. ${ }^{4}$ The NIH requests that sex as a biological variable be factored into research designs, analyses, and reporting, ${ }^{5}$ but the current lack of firm guidelines has led to a low proportion of SSA reporting in clinical trials.

Hettrich et al. ${ }^{6}$ analyzed the inclusion of SSA in articles published in 5 high-impact orthopaedic journals from 2000 to 2005 and from 2005 to 2010 . They found an increase in SSA between 2000 and 2005 but no significant increase from 2005 to 2010 . They also reported that SSA was performed in fewer than one-third of all studies included. Tisosky et al. ${ }^{7}$ evaluated SSA in all abstracts presented at the American Academy of Orthopaedic Surgeons (AAOS) annual meeting between 2006 and 2013. They found that only $5.4 \%$ of the 11,001 abstracts included adequate SSA.

The purpose of this study was to evaluate the presence of SSA in abstracts accepted for podium presentation at the Arthroscopy Association of North America (AANA) and American Orthopaedic Society for Sports Medicine (AOSSM) annual meetings from 2016 to 2019. A secondary purpose was to determine the percentage of female first or senior authors. We hypothesized that there would be a low percentage of abstracts reporting SSA, despite efforts by the NIH to include women in studies, as well as literature emphasizing the importance of its presence in clinical research. We also hypothesized that fewer first or senior authors would be women.

\section{Methods}

All abstracts accepted for podium presentation at the AANA and AOSSM annual meetings from 2016 to 2019 were reviewed by 2 independent investigators (C.H., M.M.) and evaluated for the presence of SSA. SSA was defined as the use of sex as a variable in a multifactorial statistical model. If an abstract simply included the number of male or female patients in the study but did not provide any analysis, this was not considered SSA. Cadaveric studies, animal studies, and single-sex studies were excluded from review (Table 1). Descriptive statistics were used to determine the percentage of abstracts that included SSA. Other data collected included whether the abstract had a female lead or senior author, the highest degree obtained by each female author, and the anatomic focus of the study (knee, shoulder, and so on). These variables were reported as either yes or no and entered into data collection as variable 1 or 0 . These secondary outcomes of interest included establishing whether there was any statistical correlation between SSA reporting and the presence of a female lead or senior author, which was determined using Pearson correlation coefficients. Pearson correlation coefficients were also calculated to determine whether there was an association between SSA and the anatomic focus of the study (e.g., knee, hand, or shoulder). We performed $\chi^{2}$ analysis to compare the percentage of SSA reporting with female first and senior authors with the rate of SSA reporting with male authors. $P<.05$ was selected as the threshold for statistical significance. These values were analyzed in 3 separate categories: AANA abstracts, AOSSM abstracts, and all abstracts combined.

\section{Results}

Of the 322 abstracts accepted for podium presentation at the AANA annual meeting from 2016 to 2019, 284 were selected for review after application of the exclusion criteria (Table 1). SSA was performed in 24 of these studies $(8 \%)$. Of the 708 abstracts accepted for podium presentation at the AOSSM annual meeting in this same period, 607 were reviewed; of these, 66 $(11 \%)$ reported SSA. Overall, 891 studies were analyzed $(32 \%$ from AANA meetings and $68 \%$

Table 1. Abstracts Accepted for Podium Presentation at AANA and AOSSM Annual Meetings Between 2016 and 2019

\begin{tabular}{|c|c|c|c|}
\hline & AANA & AOSSM & Combined \\
\hline No. of abstracts accepted for podium presentation & 322 & 708 & 1,030 \\
\hline No. of cadaveric, animal, and single-sex studies that were excluded ( $\%$ of total) & $38(12)$ & $101(14)$ & $139(13)$ \\
\hline No. of abstracts analyzed after application of exclusion criteria & 284 & 607 & 891 \\
\hline No. of female first authors including SSA (\%) & $5(12)$ & $12(13)$ & $17(13)$ \\
\hline No. of female senior authors including SSA $(\%)$ & $5(15)$ & $7(18)$ & $12(17)$ \\
\hline
\end{tabular}

AANA, Arthroscopy Association of North America; AOSSM, American Orthopaedic Society for Sports Medicine; SSA, sex-specific analysis. 


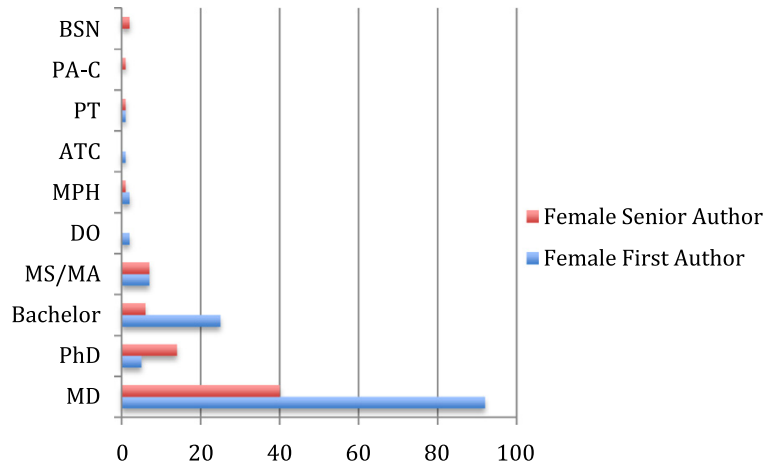

Fig 1. Degrees of female authors for all abstracts presented at Arthroscopy Association of North America and American Orthopaedic Society for Sports Medicine annual meetings between 2016 and 2019. (ATC, certified athletic trainer; DO, doctor of osteopathic medicine; BSN, bachelor of science in nursing; MPH, master of public health; MS/MA, master of science/master of arts; PA-C, certified physician assistant; PT, physical therapist).

from AOSSM meetings), with 90 abstracts (10\%) including SSA.

Of the 284 AANA abstracts, $43(15 \%)$ had female first authors and $33(12 \%)$ had female senior authors. Of the 607 AOSSM abstracts, $92(15 \%)$ had female first authors and $39(6 \%)$ had female senior authors. Altogether, 135 of the 891 total abstracts (15\%) listed a female first author and $72(8 \%)$ had a female senior author. Of the 135 female first authors, $92(68 \%)$ had an M.D., whereas 40 of the 72 female senior authors $(56 \%)$ had an M.D. Notably, there were 25 female first authors $(19 \%)$ with a bachelor's degree and 14 female senior authors (19\%) who had a Ph.D. (Fig 1).

A weak positive correlation was found between the inclusion of SSA and having a female first author (Pearson correlation coefficient $=0.048, P=.209$ ) or having a female senior author (Pearson correlation coefficient $=0.048, P=.211)$ among AANA abstracts. Female first authors of AANA abstracts reported SSA at a rate of $11.6 \%$ (Table 1 ) versus a rate of $7.9 \%$ for male first authors $(P=.423)$. Female senior authors reported SSA at a rate of $15.2 \%$ (Table 1) compared with a rate of $7.6 \%$ for male senior authors $(P=.141)$. A weak positive correlation was also found between the inclusion of SSA and having a female first author
(Pearson correlation coefficient $=0.029, P=.234$ ) or having a female senior author (Pearson correlation coefficient $=0.061, P=.066$ ) among AOSSM abstracts. Female first authors of AOSSM abstracts reported SSA at a rate of $13.0 \%$ (Table 1) versus a rate of $10.5 \%$ for male first authors $(P=.479)$. Female senior authors reported SSA at a rate of $18.0 \%$ (Table 1) versus a rate of $10.4 \%$ for male senior authors $(P=.141)$. Statistical analysis of AANA and AOSSM abstracts combined showed an overall positive correlation between reporting SSA and having a female first author, with a Pearson correlation coefficient of $0.035(P=.147)$. There was also a positive correlation between AANA and AOSSM abstracts reporting SSA and having a female senior author, with a Pearson correlation coefficient of $0.052(P=.059)$ (Table 2). Overall, female first authors reported SSA at a rate of $12.6 \%$ (Table 1 ) versus a rate of $9.7 \%$ for male first authors $(P=.304)$. Female senior authors reported SSA at a rate of $16.7 \%$ (Table 1) compared with a rate of $9.5 \%$ for male senior authors $(P=.052)$.

For each abstract, the anatomic focus of the study was identified (elbow, shoulder, foot/ankle, knee, hip, or other) (Table 2). With all abstracts combined, knee studies were found to have a statistically significant positive correlation with SSA reporting (Pearson correlation coefficient $=0.078, P=.010)$. A statistically significant negative correlation was found between SSA reporting and shoulder studies (Pearson correlation coefficient $=-0.072, P=.016)$. Although these findings were statistically significant, there may be limited clinical relevance because the Pearson correlation coefficients were negligible. There was no statistically significant correlation between SSA and any other specific anatomic focus of the studies (Table 2). Although the percentage of articles including SSA changed from year to year, there was no overall increase in reporting of SSA in abstracts accepted for podium presentation at the AANA and AOSSM annual meetings from 2016 to 2019 (Fig 2).

\section{Discussion}

From 2016 to 2019 , only $10 \%$ of abstracts accepted for podium presentation at the AANA and AOSSM

Table 2. Correlation Between SSA and Anatomic Focus of Study

\begin{tabular}{lccrr}
\hline & $\begin{array}{c}\text { No. of } \\
\text { Studies }\end{array}$ & $\begin{array}{c}\text { No. of Studies With SSA } \\
\text { (\% of Total) }\end{array}$ & $\begin{array}{c}\text { No. of Studies Without } \\
\text { SSA (\% of Total) }\end{array}$ & $\begin{array}{c}\text { Pearson Correlation } \\
\text { Coefficient }\end{array}$ \\
\hline Elbow & 54 & $3(6)$ & $51(94)$ & -0.04 \\
Shoulder & 255 & $17(7)$ & $238(93)$ & -0.07 \\
Foot or ankle & 43 & $2(5)$ & $41(95)$ & -13 \\
Knee & 344 & $46(13)$ & $298(87)$ & .01 \\
Hip & 139 & $10(7)$ & $129(93)$ & .04 \\
Other & 56 & $12(21)$ & $44(79)$ & -0.08 \\
\hline
\end{tabular}

\footnotetext{
SSA, sex-specific analysis.
} 


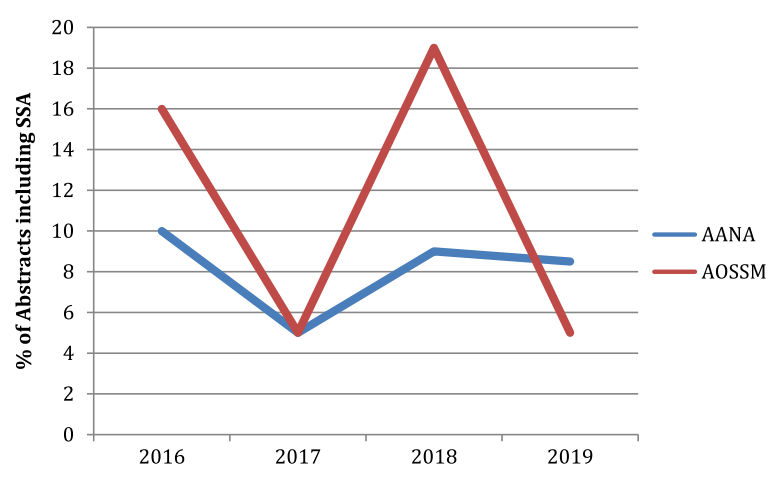

Fig 2. Percentage of abstracts including sex-specific analysis (SSA) over time (2016-2019). AANA, Arthroscopy Association of North America; AOSSM, American Orthopaedic Society for Sports Medicine.

annual meetings included SSA. AANA and AOSSM abstracts contained similar percentages of SSA reporting $(8 \%$ and $11 \%$, respectively). This finding supports our hypothesis that there would be a low percentage of abstracts reporting SSA. In addition, we found low proportions of female first authors $(15 \%)$ and female senior authors $(8 \%)$ in the abstracts analyzed.

Previous studies have similarly analyzed SSA in abstracts in the orthopaedic literature, further supporting our finding that there is a low percentage of SSA reporting. Tisosky et al. ${ }^{7}$ evaluated all abstracts presented at the AAOS annual meeting between 2006 and 2013. They found an increase in SSA reporting during this period: 48 abstracts included SSA in 2006, whereas 117 abstracts reported SSA in 2013. Their study also reported that the highest percentage of studies reporting SSA (37\%) was found within the hip and knee arthroplasty literature. However, the study showed that only $5.4 \%$ of the total abstracts presented at the AAOS annual meeting between 2006 and 2013 included SSA. Additionally, Hettrich et al. ${ }^{6}$ found that there was an increase in SSA reporting in high-impact orthopaedic journals from 2000 to 2010 , but overall, fewer than one-third of studies included SSA. Similarly, a study by Gianakos et al. ${ }^{8}$ found that $30.5 \%$ of studies in 3 high-impact orthopaedic sports medicine subspecialty journals included SSA. Furthermore, another study by Gianakos et al. ${ }^{9}$ evaluated all articles published in 6 leading orthopaedic journals in 2016. The authors found that $34 \%$ of studies included sex as a variable in a multifactorial statistical model. Of the studies reporting SSA, 39\% found a difference in outcomes between male and female patients. ' This finding shows the importance of SSA reporting in the orthopaedic literature.

A study by Xiao et al. ${ }^{10}$ evaluated the distribution of male versus female authors among all original articles from Annals of Surgery, American Journal of Surgery, JAMA Surgery, The Journal of Surgical Research, and Surgery from 2011 to 2012. The authors showed that
$70.3 \%$ of first authors were men and $84.6 \%$ of senior authors were men. Studies with female authors included a higher median number of female study participants. In addition, an interesting finding of this study was that the authors of articles with higher percentages of sex matching of participants received more citations of their work. Xiao et al. concluded that there is prevalent sex bias in surgical research, but studies that address this bias were rewarded with increased citations. In our study, 15\% of first authors were women and $8 \%$ of senior authors were women. Studies with female first or senior authors included SSA at a higher rate than studies with male first or senior authors. This analysis also found that most female authors had an M.D. One major contributing factor to the discrepancy in the number of female versus male authors in the orthopaedic literature is that there are far fewer female orthopaedic surgeons. As of the 20162017 academic year, only 14\% of orthopaedic surgery residents were women; women also only represent $6.5 \%$ of AAOS members. ${ }^{11}$ In a brochure distributed for the 2019 AANA annual meeting, AANA reported that it has a $10 \%$ female membership rate. ${ }^{12}$ Promotion and inclusion of women in orthopaedics are important for many reasons, and this study shows that one association of female authorship in clinical research is a higher percentage of SSA reporting. This, in turn, benefits patients because it contributes to a greater understanding of how to assess and treat sex differences in orthopaedics. A study by Hiller et al. ${ }^{13}$ analyzed 3 highimpact orthopaedic journals and found that there has been an increase in the percentage of female first authors (from $11 \%$ in 2006 to $17 \%$ in 2017).

\section{Limitations}

There are several limitations to this study. First, only the abstracts accepted for podium presentation at the AANA and AOSSM annual meetings between 2016 and 2019 were available for review. It is possible that some studies performed SSA but did not report it in the abstract. Additionally, there was a small sample size. Even with combining data from the AANA and AOSSM, there were still only 90 abstracts that reported SSA. Many abstracts were also eliminated from analysis because they were cadaveric, animal, or single-sex studies. Moreover, this study only analyzed abstracts presented over a 4-year period, which contributed to the small sample size. Therefore, when looking at correlations between SSA and other factors such as female authors or the anatomic focus of a study, the chances of statistical significance were less. The 4-year period evaluated in this study (2016-2019) was not compared with an earlier period; thus, it is not possible to know if there was a change in SSA reporting between 2 different periods. Regarding the authorship analysis, listing of the first or senior author is sometimes not 
reflective of the contribution the author truly made, so this may be a limitation to the analysis between female authorship and SSA inclusion. Finally, no guideline has been established for determining when SSA is necessary and appropriate in statistical analysis. SSA may not be practical for all studies. It is currently unclear which studies should include SSA.

\section{Conclusions}

From 2016 to 2019 , only $10 \%$ of abstracts accepted for podium presentation at the AANA and AOSSM annual meetings included SSA. Altogether, women represented $15 \%$ of first authors and $8 \%$ of senior authors.

\section{References}

1. Cheung EC, Boguszewski DV, Joshi NB, Wang D, McAllister DR. Anatomic factors that may predispose female athletes to anterior cruciate ligament injury. Curr Sports Med Rep 2015;14:368-372.

2. Wentz L, Liu PY, Haymes E, Ilich J. Females have a greater incidence of stress fractures than males in both military and athletic populations: A systemic review. Mil Med $2011 ; 176: 420-430$.

3. Alswat KA. Gender disparities in osteoporosis. J Clin Med Res 2017;9:382-387.

4. National Institutes of Health, Office of Extramural Research. NIH policy and guidelines on the inclusion of women and minorities as subjects in clinical research. Bethesda, MD: Department of Health and Human Services, National Institutes of Health, 2001.

5. National Institutes of Health, Office of Research on Women's Health. NIH policy on sex as a biological variable. https://orwh.od.nih.gov/sex-gender/nih-policysex-biological-variable. Accessed November 13, 2019.

6. Hettrich CM, Hammoud S, LaMont LE, Arendt EA, Hannafin JA. Sex-specific analysis of data in high-impact orthopaedic journals: How are we doing? Clin Orthop Relat Res 2015:473:3700-3704.

7. Tisosky A, Logan C, Brook E, Xu J, Matzkin E. Reporting the influence of sex in research: Trends at AAOS annual meetings. J Am Acad Orthop Surg 2019;27:112-117.

8. Gianakos AL, Szukics P, George N, Elkattawy S, LaPorte DM, Mulcahey MK. Sex-specific analysis at two time points in three high-impact orthopaedic sports medicine journals. Arthrosc Sports Med Rehabil 2020;2: e207-e212.

9. Gianakos AL, George N, Pinninti A, Kwan S, LaPorte D, Mulcahey MK. CORR insights: Sex- and gender-specific analysis in orthopaedic studies. Clin Orthop Relat Res 2020;478:1489-1490.

10. Xiao N, Mansukhani N, Mendes de Oliveira D, Kibble M. Association of author gender with sex bias in surgical research. JAMA Surg 2018;153:663-670.

11. Chambers CC, Ihnow SB, Monroe EJ, Suleiman LI. Women in orthopaedic surgery: Population trends in trainees and practicing surgeons. J Bone Joint Surg Am 2018;100:el16.

12. Arthroscopy Association of North America. AANA19 annual meeting Orlando. https://www.aana.org/ aanaimis/sitedownloads/education/annualmeeting/2019/ exhibitor/prospectus.pdf. Accessed November 13, 2019.

13. Hiller K, Boulos A, Tran M, Cruz A. What are the rates and trends of women authors in three high-impact orthopaedic journals from 2006-2017? Clin Orthop Relat Res 2020;478:1553-1560. 